\title{
REVUE GÉNÉRALE.
}

\section{SUR L'ANAPHYLAXIE LAGTEE,}

PAR

\author{
M. PÉHU, et
}

Médecin de la Charité de Lyon.
P. BERTOYE,

Interne des Hôpitaux

Depuis une quizaine d'années la question de l'anaphylaxie lactée en pathologie humaine a été l'objet de nombreuses recherches. La curiosité des médecins a été éveillée sur elle pour de multiples raisons. La première est que cette question présente indubitablement un grand intérêt pratique : il s'agit en effet de rechercher si des troubles, à évolution bien déterminée, constituent une manifestation du type anaphylactique tel qu’il a été décrit en 1902 par Richet ; la clinique humaine et, particulièrement, la médecine infantile sont intéressées à là solution du problème. D'autre part, à ce sujet, est attachée une importance pratique : ear il est fort intéressant de préciser dans quelles limites un aliment normal, d'origine humaine ou animale, dont la destinée n'est théoriquement qu'utile, peut devenir nuisible pour l'organisme dans lequel il est introduit.

I.

D'un point de vue historique, il convient d'établir que Maurice. Anthus en 1903 découvrit le pouvoir anaphylactogène du lait : ce que signifie cette constatation, nous le préciserons ultérieurement. Mais 11 serait injuste de refuser à $M$. Antrus la priorité de cette découverte.

Elle resta pendant plusieurs années dans le domaine des connaissances théoriques. C'est en 1908 que M. Hutinel s'efforça de montrer la nature anaphylactique de certains troubles digestifs observés dans l'enfance. L'éminent pédiâtre publie quelques observations démonstratives pour l'explication desquelles il semblait en effet rationnel d'invoquer le mécanisme d'une hypersensibilité digestive succédant à l'évolution d'une gastro-entérite infantile. Dès ce moment, la question est discutée dans divers pays. En France, H. Barbier l'étudie dans un mémoire documenté (1910). Puis M. Terrien en discute la réalité (1911), du moins quand il s'agit de l'intolérance pour le lait féminin. 
Dans les pays de langue allemande, à plusieurs reprises déjà, le sujet avait fait l'objet de recherehes cliniques et expérimentales parmi lesquelles il faut citer principalement : Finkelstein (1905), SAlge (1906), Zybel (1910), Wernstedt (1910), Halberstadt (1911). Le mémoire très complet de Kleinschmidt (1913) expose des expériences exéeutées sur des cobayes. Plus tard on peut encore citer des articles de Stricker (1920), de Rhonheimer (1921). En Italie Finizio (1911) publie un mémoire important et documenté sur la question : il l'intitule “L'anaphylaxie familiale pour le lait de vache». M. Pesci (1916) consacre à cette question quelques pages intéressantes dans son récent livre sur les formes anaphylactiques. Les médecins américains, surtout dans ces dernières années, ne sont point restées indifférents à ce sujet; on trouvera d'intéressants détails dans les travaux de F.-B. Talbot, Hoobler (1916), Walker, Schulz et Larson (1918.

Quelques revues d'ensemble lui ont été consacrées, en particulier, celle de M. Weill-Halle publiée en 1914 par le journal Le Nourrison. Dans son récent livre surl'Allaitement 1920 , M. Marfan,avec raison, réclame une délimitation exacte du domaine de l'anaphylaxie : " en raison de l'extraordinaire extension donnée récemment à « eet état, il faut, objecte-t-il, qu'on nous dise comment on le définit, " et à quoi on le reconnait ».

Cet essai de délimitation est certainement nécessaire. D'autre part, certaines méthodes, récemment préconisées, permettent d'élargir le problème de l'anaphylaxie lactée : nous voulons parler des injections sous-cutanées ou intra-veineuses de lait en médecine humaine. Notre dessein n'est pas de les envisager dans leurs résultats pratiques ; nous voulons seulement, de leur emploi, tirer des déductions touchant le problème de l'anaphylaxie lactée ; car l'introduction " parentérale » du lait nous permet désormais d'envisager comparativement les influences nuisibles du lail suivant qu'il pénètre dans l'organisme par cette voie anormale, ou, au contraire, par le tube digestif.

La physiologie expérimentale a apporté,de son côté, quelques documents au débat. La première constatation du pouvoir anaphylactogène du lait a été faite, nous l'avons dit, par M. Arthus (1903); puis cette propriété a été l'objet d'études entreprises par Bartenstein (1905), Rosenau et Anderson, Besredka (1909). Puis, ce sont les recherches entreprises systématiquement en France pour réaliser l'anaphylaxie alimentaire par Lesné et Dreyrus, Barnathan, Ch. Riamet père, puis Guy Laroche, Ch. Richet fils et Saint Girons, en Allemagne par Kileinschmidt. Enfin, tout récemment, M Pentimat. (1921) enireprend chez le lapin des expériences pour déterminer le pouvoir toxique du lait en injections intra-veineuses réitérées. 
La pathologie des troubles spontanés peut être envisagée de la façon suivante.

Etiologiquement, on relève que les cas appartiennent, en général, à la première enfance. Il n'est pas très fréquent d'observer ces phénomènes morbides dans un àge plus avancé de la vie. Toutefois cette proposition n'est que partiellement vraie, car on peut noter que des enfants déjà grands et des adultes présentent, d'une façon persistante, des phénomènes d'intolérance si prononcée pour le lait que, sauf l'âge, nulle différence ne sépare la symptomatologie de celle qui est relevée chez le nourrisson. Un autre caractère commun est que, le plus souvent, il s'agit de lait de vache. Celui-ci est le grand facteur des troubles. Les autres laits d'animaux - peut-être parce que rarement employés, ou pour d'autres raisons qui nous échappent - ne sont guère des agents producteurs de ces troubles : lait d'ânesse, de chèvre ou de jument ne figurent pas à l'actif de ces manifestations pathologiques.

Le lait de femme peut exceptionnellement les produire. En vérité les observations, démonstratives, de cette catégorie sont fort rares. Dans son traité de l'allaitement (1920), M. MARFan (page 442) cite les cas publiés : il arrive à la conclusion que les cas d'intolérance des nourrissons pour le lait de femme ne sauraient être révoqués en doute, mais qu'ils sont exceptionnels. Plusieurs auteurs ont fait remarquer que. dans ıes cas authentiques d'anaphylaxie par et pour le lait de femme, il parait bien difficile d'invoquer l'action perturbatrice d'une albumine hétérogène.

Qu'il s'agisse d'intolérance lactée congénitale ou acquise, le passé pathologique des malades est souvent chargé. On mentionne par exemple : de la suralimentation causant des désordres latents, peutêtre des lésions parenchymateuses ; la naissance avant terme ; des maladies générales, en particulier tuberculose ou spécificité auxquelles M. Barbier attache une grande importance; de l'hérédité nerveuse ou neuro-arthritique, la diathèse exsudative. La recherche des antécédents héréditaires ou familiaux ne doit point être négligée; elle fournit parfois des renseignements utiles ; elle montre, par exemple, une intolérance des ascendants vis-à-vis du lait de vache, ou encore, père et mère éprouvent à l'endroit de cet aliment une répulsion souvent profonde.

Sur le terrain clinique, il convient de distinguer deux variétés principales de manifestations, 
Dans certains cas, relativement fréquents, les phénomènes surviennent au cours des premières années de la vie, particulièrement dans la période de l'alimentation lactée exclusive, ou encore à l'époque du sevrage c'est-à-dire de 8 à 15 mois ; ces chiffres étant, cela va sans dire, assez approximatifs. Ils ne se manifestent pas dès les premiers temps de l'allaitement ; des conditions spéciales, que nous pré- ciserons ultérieurement, doivent être réalisées pour les déterminer. Un second groupe comprend les cas où, dès la première ingestion du lait, apparaissent des symptômes sévères ou graves. C'est à ces faits qu'a été appliqué le terme d'idiosynerasie.

$I^{\text {er }}$ groupe. - Il comprend, nous le répétons, les observations dans lesquelles les symptômes apparaissent après une période plus ou moins longue d'alimentation lactée. D'excellentes études ont été publiés sur ce sujet.

a) M. Hutinel a appelé l'attention sur les faits suivants. Il s'agit d'enfants ayant présenté antérieurement des troubles digestifs à allure le plus souvent aiguë : vomissement et surtout diarrhée, gastroentérite, entérocolite grave, ayant conduit à l'interdiction du lait pendant une période variant de quelques jours à quelques semaines.

Dès la reprise, cependant prudente, du lait de vache, on observe des phénomènes particulièrement graves, suivant de quelques minutes l'ingestion de l'aliment perturbateur : vomissements répétés, en même temps que diarrhée abondante, glaireuse, état général sévèrement atteint, collapsus plus ou moins profond, avec faiblesse du pouls, oligurie, et même, dans certains eas troubles nerveux surajoutés (convulsions, état spasmodique des extrémités, strabisme, perte de connaissance).

Des éruptions urticariennes ou érythémateuses peuvent apparaittre sur le visage, les bras ou les jambes. On observe le plus souvent une répercussion manifeste sur l'état général : les yeux s'excavent, le nez se pince, la face prend un aspect blafard, le teint présente une coloration plombée.

Les phénomènes aigus à allure impressionnante ont une durée moyenne de quelques jours. Puis, le plus souvent, ils s'atténuent et disparaissent Mais la réaccoutumance du lait de vache n'est pas facilement réalisée ; il faut observer une grande prudence dans l'alimentation. La terminaison fatale est fort rare. Seule dans le cas de Finkelstein (Observation IV de son mémoire de 1905̈) on a malheureusement enregistré un décès. Cette observation peut ètre citée comme un exemple d'aggravation progressive des symptomes à chaque tentative nouvelle de réalimentation par le lait de vache; et quand, un dernier essai, " témoignant d'une persévérance regret- 
table, est tenté, la mort survient dans le collapsus 》(1). L'autopsie a été pratiquée : elle ne montra aucune lésion caractéristique en dehors d'une légère entéro-colite séro-hémorrhagique; on constata l'existence d'une infiltration légère des reins et de la stéatose hépathique. Ces lésions n'ont rien de pathognomonique; elles témoignent d'altérations toxiques des principaux viscères.

b) A eôté de ces phénomènes si caractérisés, on peut placer d'autres manifestations, celles-là, beaucoup plus fréquentes, à caractère plus estompé, et qui semblent cependant ressortir à l'intolérance, progressivement réalisée, du nourrison pour le lait de vache.

M. Barbier (1910) en a fourni une analyse clinique très minutieuse : on peut les appeler " troubles de la petite anaphylaxie » tandis que les accidents aigus décrits dans le paragraphe précédent seront dénommés « «la grande anaphylaxie ». M. BARBier signale qu'ils sont, en général, précédés par une phase, latente ou larvée, dans laquelle, d'abord, le poids augmente d'une façon excessive; puis, dans une deuxième période, il reste stationnaire ; enfin, lorsque les accidents digestifs apparaissent (vomissements, diarrhée, etc), il baisse progressivement jusqu'à ce que l'on se décide à supprimer pendant une période plus ou moins longue, l'usage du lait.

2. Groupe.- Il est constitué par les cas dans lesquels les symptômes surviennent, dès la première ingestion du lait, particulièrement le lait de vache. C'est à ces faits qu'on appliqué le terme d'idiosyncrasie. De nombreux exemples en ont été cités; on peut rappeler ceux de Finızıo, particulièrement les Observations I et II de son mémoire : elles concernent deux frères (non jumeaux) qui tous deux présentaient des signes d'intolérance dès la première ingestion. La symptomatologie de ces cas est encore mieux tranchée que pour ceux de la première catégorie, et sâns anticiper sur les conclusions qui sont tirées de cette constatation, nous signalerons que la plupart des auteurs ont été frappés par la similitude symptomatique de ces manifestations avec celles du choc anaphylactique.

En général, le tableau morbide apparaît dès la première ingestion de lait de vache, alors qu'on ne peut en aucune façon invoquer le rôle d'une sensibilisation antérieure, du moins, une influesee non congénitale. Les troubles sont provoqués par l'ingestion d'une quantité minime de lait variant de 10 à $100 \mathrm{gr}$., mais qui est toujours peu considérable. Il va de soi qu'on ne saurait, en pareil cas, songer à incriminer la composition chimique ou biologique du lait. Les phénomènes apparaissent avec rapidité, quelques minutes après la péné-

(1) Texte de MM. Guy Laroghe, Ch. Richet et Fis et F. Saint-Girons.L'anaphylaxie alimentaire. Actualités Médicales, chez Bailliêre (1919, page 30). 
tration du lait dans les voies digestives : c'est à dessein que nous employons ce terme général; car si, le plus souvent, la pénétration a lieu par l'estomac, des phénomènes peuvent être déterminés, comme dans un cas de M. Hutinel, par un lavement de lait.

Les phénomènes, en général, se déroulent dans un ordre défini : vomissements, diarrhée liquide, verdâtre, séreuse, parfois sanguinolente avec tenesme rectal ; manifestations du système nerveux (hypertonie, tétanie, convulsions) ; dyspnée; modifications de la température, en général hyperthermie ; souvent éruptions cutanées du type urticarien avec tuméfaction du visage, saillie considérable des lèvres, succédant de quelques minutes à l'ingestion du lait : elle est signalée dans les observations de Striker (1920), de Rhonheimer (1921) : et ce dernier auteur ajoute que la ressemblance des phénomènes morbides, particulièrement des éruptions, est si grande que l'on peut, sans hésitation, dans ces cas, admettre une réaction anaphylactique.

Malgré le caractère impressionnant de ces réactions au lait de vache, la terminaison des incidents aigus, marquant la pénétration de cet antigène, est le plus souvent favorable. Quelques cas de mort out été cités. Ils sont, avons-nous dit, très rares. Nous avons rapporté l'observation de Finkelstein. Dans le cas 3 du travail de Finızıo (mère tuberculeuse), une première tentative d'alimentation lactée faite, à une date non indiquée dans le texte, avec $120 \mathrm{gr}$. de de lait de vache, avait provoqué au bout de 2 à 3 heures des vomissements, une diarrhée abondante et de la dyspnée. Ultérieurement, les mêmes symptômes apparurent, atténués, consécutivement à l'ingestion de farine lactée ou de babeurre. Mais au soixantième jour de la vie de l'enfant on essaie à nouveau $100 \mathrm{gr}$. de lait: des phénomènes d'une intensité particulière se montrèrent (au bout d'un temps non préciséj et la mort s'en suivit. L'autopsie ne fut pas pratiquée. Un autre cas mortel a été observé par Halberstadt (cas III de son mémoire de 1911) : il s'agissait d'un nourrisson dont le jumeau présentait également une intolérance lactée manifeste. L'auteur dit que l'autopsie n'a fourni aucun renseignement.

En général, soit par une atténuation spontanée, soit parce que, progressivement, on parvient à réaliser chez les sujets une désensibilisation, une réaccoutumance, les troubles s'atténuent et disparaissent. Il est plutôt excẹtionnel qu'ils persistent après la fin de la deuxième année : en compulsant les observations, on relève que la susceptibilité d'abord très marquée des nourrissons ne persiste pas. Plusieurs 
médecins affirment n'avoir jamais observé l'intolérance complète et définitive d'un enfant au lait de vache.

\section{III.}

Pour mieux permettre l'incorporation dans l'anaphylaxie de ces cas à symptomatologie bien tranchée, on s'est efforcé de déceler en eux les critères de cet état spécial, soit en dehors de toutes manites tations cliniques, soit au moment des symptômes aigus, traduisant le choc humoral.

$1^{\circ}$ Dans l'anaphylaxie non « extériorisée », plusieurs tests ont été recherchés :

a) On a voulu déterminer la présence d'une albumine étrangère dans le sang des nourrissons. Or l'albumine étrangère ne peut être que celle du lait de vache. Quelques résultats positifs ont été enregistrés. Tesdeschi, Salge, Bauer et Moro, cités par Pesci, chez des enfants atrophiques avec troubles digestifs, la décèlent dans le sang circulant par le moyen de la réaction précipitante. Ganghofner et Lange concluent dans le même sens. Neuhaus, Kleinschmid par le moyen d'un anti-sérum affirment avoir démontré la présence d'une albumine bovine dans le sang des nourrissons anaphylactisés par et pour le lait de vache. Cette question est d'ailleurs traitée dans le mémoire de Finizio.

De toute façon, il convient d'être très réservé sur la valeur de cette recherche. Si les résultats sont positifs, il faut encore,pour leur accorder une valeur probante, que les enfants sur qui la recherche a été faite présentent parallèlement des signes authentiques d'anaphylaxie lactée. Si, d'autre part, on ne décèle pas la présence de l'albumine étrangère, cela ne signifie pas qu'elle ne pénètre pas dans la circulation, car cette pénétration peut s'accomplir très passagèrement et n'être pas décelée par nos méthodes habituelles d'examen. D'ailleurs, comme le remarque avec raison Finızıo, les précipitines et les anticorps anaphylactiques sont des substances différentes. Entre la quantité de précipitines contenues dans le sérum et son pouvoir anyphylactogène il n'existe pas de rapport direct, notion établie par les recherches de Doenr et Russ, de Friedmann, de Scott (1909).

b) Quelques auteurs ont cherché à provoquer l'anaphylaxie passipe par injection de sang du malade à un animal. Les résultats ont été irréguliers et inconstants. Ноовцer (1916) inocule, dans le péritoine d'un cobaye, lu sang prélevé chez des nourrissons qui présentaient de l'intolérance lactée. Onze jours après, ces animaux reçoivent également, par voie péritonéale, $3 \mathrm{cc}$. de lait : il produit ainsi des choes anaphylactiques chez des animaux. Schultz et Larson (1918) sous 
traient un peu de sang chez des nourrissons atrophiques suralimentés par des doses exagérées de lait, ou atteints de vomissements périodiques ou encore de diathèse exudative. Ils injectent le sang de ces petits malades à des cobayes qu'ils éprouvent ultérieurement par du lait. Les résultats obtenus sont également irréguliers : seulement dans le $4^{\circ}$ groupe, ils provoquent des phénomènes anaphylactiques.

De mème l'anaphylaxie passive n'a pas été réalisée par Moro et Witzincer. Il semble donc que cette épreuve n'ait pas une très grande valeur. D'ailleurs, comme le remarque Kreinschmint, il n'est guère commode de l'appliquer dans la pratique çourante.

c) A l'heure actuelle, on recherche beaucoup à utiliser pour le diagnostic de certains états anaphylactiques, les réactions cutanées à l'aliment ou à la substance incriminé.

L'exemple a été fourni par ceux qui ont préconisé la méthode épidermique ou dermique pour déterminer l'état d'hypersensibilisation au sérum thérapeutique après inoculation sous-cutanée de ce dernier. Pour établir la sensibilisation au lait de vache, les recherches ont été nombreuses.

Mais les résultats ne sont guère concluants: ScHмrdт (1910), Finizio (1911), Kleinschmidt (1913), emploient le lait cru, ou bouilli, ou chauffé à $ّ 6$ pendant une $1 / 2$ heure à 1 heure, et l'introduisent par voie intra-dermique. D'après eux, on ne peut, en aucune façon, reconnaître, par une réaction cutanée ainsi pratiquée, l'état anaphylactique. Toutefois des médecins américains, M. ТАLвот, de Boston, par exemple, préconisent la cuti-réaction par l'incision faite à la peau de l'avant-bras ; s'il existe de l'hypersensibilité au lait de vache, on constate l'existence d'une papule urticarienne manifeste au bout de 2 à 10 minutes; elle disparait au bout d'une demi-heure à 2 heures. On a cherché à provoquer les réactions de la peau, au moyen de caséine sèche de lait de vache, avec du sérum de bouf(Schlossmann). Mais ces expériences n'ont pas une valeur très grande, car on n'est pas en droit d'établir une analogie rigoureuse entre les albumines du sérum sanguin ou d'une des parties constituantes du lait, d'une part, et le lait complet ingéré, d'autre part.

Sur ce point spécial des réactions cutanées (cuti ou intradermo) obtenues par le lait de femme ou de vache, nous pouvons apporter quelques documents personnels : l'un de nous (P. Bertoye) a collaboré aux intéressantes recherches du professeur Weitr dont nous parlerons bientôt. Un nombre important d'intra-dermo-réactions ont été pratiquées avec ces deux laits sur des enfants de 0 à 2 ans, normaux ou porteurs d'affections diverses, ou encore, atteints de maladies digestives. Or, certains nourrissons dyspeptiques présentent des intra-dermo réactions positives; quelques uns, bien portants, en 
montrent également. Nulle règle constante ne peut être déduite. De ces recherches nous avon; tiré eette impression qu'il est bien difficile de dégager des conclusions précises, en s'appuyant sur le earactère positif ou négatif de l'intra-dermo réaction au lait de vache ou de femme, pour déceler à coup sûr la sensibilisation, à ces aliments, des nourrissons.

$2^{\circ}$ An moment des accidents aigus, que leur ensemble symptomatique permet de comparer au choc anaphylactique, il n'a pas été facile de rechercher systématiquement l'état du sang. On peut d'ailleurs à ce point de vue, observer que des études hématologiques ont été poussées loin dans certains syndrômes, tels que l'asthme, par cette simple raison qu'il a été souvent facile de les provoquer délibérément au gré de l'observateur: les recherches de M. Widal et de ses collaborateurs sont significatives à cet égard. Par contre, la crise révélatrice du déséquilibre sanguin, appelée par $M$. Widal crise hémoclasique, et suscitée par certaines substances comme les arsénobenzènes, n'a pas été étudiée en détail. Cela se comprend aisément, si l'on veut réfléchir que ces manifestations aiguës apparaissent soudainement et ne laissent guère à l'observateur le temps d'en saisir avec exactitude le mécanisme. Il en est de même pour les cas de grande anaphylaxie lactée : et c'est pourquoi sans doute, on trouve peu à glaner dans la littérature médicale touchant la crise hémoclasique dans ces états d'intolérance observés chez le nourrisson.

Les documents fournis dans ce domaine sont done rares et peu concluants. Seul, M. Finizio, dans les deux cas II et IV, a pu, dans le sang des nourrissons, au moment même où ils ont présenté des symptômes aigus (vomissements, diarrhée, collapsus, troubles nerveux) déceler la présence de l'albumine hétérogène.

Pour les mêmes raisons, sans doute, la numération des leucocytes n'a pas été faite systématiquement. Au surplus, des études sur lesquelles nous ne voulons point insister, et qui sont bien résumées dans la thèse de M. Langle faite sous l'inspiration de M. Lesné (1921), celles de M. Dorlencourt (1920-1921; montrent quelle réserve il faut apporter dans la recherche de la leucocytose digestive chez le nourrisson.

\section{IV.}

Au cours de ces derniêres années, on a beaucoup employé, en médecine humaine, un nouveau mode d'inoculation du lait dans l'organisme : les injections sous-cutrnées. Celles-ci ont été utilisées dans trois ordres de cas bien distincts. Quelques auteurs, en France, en Allemagne, en Autriche, en ont préconisé l'emploi dans diverses 
maladies : infections générales, fièvre typhoïde, grippe (SснміDт (1916), Muller, THirolorx (1918); dans les maladies oculaires (Domec, de Dijon, L. Dor!. Quelques années auparavant, M. Nolf (de Liège) en avait préconisé l'emploi (1911) pour stimuler la sécrétion lactée chez les nourrices médiocres. Enfin, depuis 3 années environ,le professeur Weill a préconisé le traitement de certaines formes d'intolérance lactée chez le nourrisson par les injections sous-cutanées du lait non toléré. A l'origine de ses recherches,M. WeILl s'était laissé guider par les notions admises sur les états anaphylactiques, pour imaginer la méthode qu'il préconise et qui, judicieusement appliquée, fournit des résultats excellents. A ces yeux cependant, il ne faut pas établir une identité entre l'anaphyxalie et l'intolérance, car, ainsi qu’il le fait remarquer, en général « la désensibilisation par l'emploi de petites doses d'antigène, proposé par B BSREKA, est temporaire, tandis que l'immunité contre l'intolérance obtenue par les injections de lait, est durable m.

Quoi qu'il en soit, entreprises dans des buts différents, ees méthodes offrent des enseignements qu'on peut utiliser d'un point de vue général. En effet, des injections premières de lait par voie sous-cutanée peuvent entraîner des accidents locaux à apparition plus ou moins rapide. Ces accidents consistent dans des rougeurs ou des phénomènes fluxionnaires de la peau, des adénopathies. Parfois même, on observe des accidents généraux tels que : fièvre, nausées, collapsus. Il est vraisemblable qu'il s'agit alors de manifestations dues à la toxicité primaire du lait, manifestations qui ne s'écartent guère de celles provoquées dès l'introduction première, par voie hypodermique ou veineuse, des sérums.

Il en est de même des effets locaux ou généraux produits par les réinjections sous-cutanées et même intra-veineuses de lait. Assez fréquemment, et contrairement à l'affirmation de SснміDт, elles peuvent produire des choes anaphylactiques : telle l'observation de Lubliner concernant une jeune fille de 18 ans chez qui une réinjection de $10 \mathrm{ccs}$. de lait, pratiquée au bout de 8 jours, détermina l'apparition d'un choc anaphylactique grave ; telle encore l'observation de O. Sachs (1918), également démonstrative. Comme dans les réinjections de sérums curateurs, il n'est pas obligatoire que survienne un choe anaphylactique plus ou moins grave ; mais cette éventualité est possible, et cela ne doit pas surprendre, car il s'agit d'une injection d'une substance protéique amenant d'une façon presque mathématique des modifications humorales et celulaires de l'organisme, c'est-à-dire un état anaphylactique. 
En comparant ces deux ordres de manifestations symptomatiques, les résultats observés dans l'introduction de laits par voie digestive ou parvoie " parentérale »; on peut done tirer les conclusions suivantes :

1. L'absorption uniquement par les ooies digestipes de différents laits peut, dans des circonstances déterminées, propoquer des symptômes rappelant i'anaphylaxie locale ou générale; mais, en fait, ces cas sont plutôt exceptionnels, au regard de la fréquence avec laquelle on observe, surtout en médecine infantile, des troubles digestifs banals ne présentant en aucune façon des caractères anaphylactiques.

2. Introduit uniquement par voie "parentérale», sous-cutanée ou mieux intra-seineuse, le lait peut engendrer une sensibilisation, un état anaphylactique, prouvé par l'apparition d'un choc lors de l'injection seconde.

3. Au contraire, des injections sous-cutanées de lait, arant été faites pendant une période suffisante pour engendrer l'état d'anaphylaxie, l'introduction ultérieure de lait, par les poies digestipes, ne propoque pas de choc.

M. Weill qui a pratiqué une série d'injections sous-cutanées de lait chez des nourrissons, pour traiter des troubles digestifs, n'a jamais observé une sensibilisation; l'injection consécutive du lait n'a produit aucune manifestation anaphylactique.

(A suipre).

\section{BIBLIOGRAPHIE ANALYTIQUE}

\section{$1^{\circ}$ LIVRES.}

Donntc. - Le contrôle du lait. $\Psi^{\mathrm{e}}$ édition. Petite bibliothèque agricole. J.-B. Baillière et fils, $4 \mathrm{fr}$.

C'est dans la seconde moitié du XIXe siècle que les méthodes scientifiques ont permis, pour la première fois, d'aborder l'étude du contrôle chimique et physique du lait. A côté des noms de Donaḱ, Bouchardat, Quévenne, Marchand, Аdam, etc., nous rencontrons ceux, plus modernes, de Вавсоск, de Gerber et de Dornic.

Depuis Pune qui conseillait de verser une goutte de lait sur l'ongle et de n'admettre comme pur que le lait qui « in ungue hæret nee defluit », il faut reconnaître que l'on n'avait rien pu faire, jusqu'à cette époque, si ce n'est de substituer à l'ongle une aiguille à tricoter qui, plongée dans le lait, puis 